\title{
Factores sociales que influyen en aumentar el contagio de la covid-19 en México
}

\section{Social Factors that Influence in Increasing the Spread of COVID-19 in Mexico}

Fatores sociais que influenciam o aumento da propagação da covid-19 no

México

Martha Jiménez García

Instituto Politécnico Nacional, Unidad Profesional Interdisciplinaria de Ingeniería y

Ciencias Sociales y Administrativas, México

majimenez@ipn.mx https://orcid.org/0000-0002-8556-2955

Pilar Gómez Miranda

Instituto Politécnico Nacional, Unidad Profesional Interdisciplinaria de Ingeniería y

Ciencias Sociales y Administrativas, México pgomezm@ipn.mx http://orcid.org/0000-0002-1480-3061

Maria Elena Tavera Cortes

Instituto Politécnico Nacional, Unidad Profesional Interdisciplinaria de Ingeniería y Ciencias Sociales y Administrativas, México mtavera@ipn.mx https://orcid.org/0000-0002-2179-2735

Ma. De los Ángeles Martínez Ortega Instituto Politécnico Nacional, Unidad Profesional Interdisciplinaria de Ingeniería y

Ciencias Sociales y Administrativas, México mmartinezo@ipn.mx https://orcid.org/0000-0002-8483-9844 


\section{Revista Iberoamericana para la Investigación y el Desarrollo Educativo ISSN 2007 - 7467}

Francisco Pérez Soto

Universidad Autónoma Chapingo, México

perezsotofco@gmail.com

http://orcid.org/0000-0002-7982-420x

\section{Resumen}

El objetivo de este trabajo de investigación fue analizar los indicadores de salud y de pobreza que influyen en el aumento de contagios por el coronavirus de tipo 2 causante del síndrome respiratorio agudo severo (SARS-CoV-2) a través de dos modelos predictivos. Se realizó un análisis en 2409 municipios de México en dos periodos de tiempo. Para el periodo dos (13 de julio del 2021) las variables "Neumonía”, “Asma”, “Otra complicación”, “Obesidad” y "Otro caso" influyeron en el contagio de covid-19 en 0.4024, 0.5229, 2.4246, 1.0053 y 1.4788, respectivamente. De igual forma, las carencias de ingresos, vivienda y alimentación fueron variables sociales que influyeron en el número de contagios, debido a que no se pudo mantener "sana distancia". Se concluye con la demande de que el Gobierno aplique mayores medidas tanto de salud como de conciencia social al contagio del covid-19, así como de programas sociales en los lugares donde existan personas vulnerables por ingresos, personas con carencia en calidad y espacios de vivienda y personas con carencia en acceso a la alimentación.

Palabras clave: covid-19, pandemia, pobreza, salud, vivienda.

\section{Abstract}

The objective of this research work was to analyze the health and poverty indicators that influence the increase in infections by the type 2 coronavirus that causes severe acute respiratory syndrome (SARS-CoV-2) through two predictive models. An analysis was carried out in 2409 municipalities of Mexico in two periods of time. For period two (July 13, 2021) the variables "Pneumonia", "Asthma", "Other complication", "Obesity" and "Other case" influenced the spread of covid-19 in 0.4024, 0.5229, 2.4246, 1.0053 and 1.4788, respectively. Similarly, the lack of income, housing and food were social variables that influenced the number of infections, due to the fact that a "healthy distance" could not be maintained. It concludes with the demand that the Government apply greater measures of both health and social awareness to the contagion of covid-19, as well as social programs in 


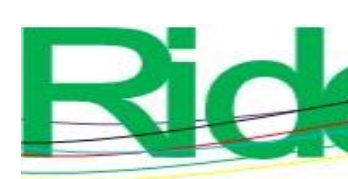

Revista Iberoamericana para la
Investigación y el Desarrollo Educativo ISSN $2007-7467$

places where there are vulnerable people due to income, people with a lack of quality and housing spaces. and people with lack of access to food.

Keywords: covid-19, pandemic, poverty, health, housing.

\section{Resumo}

O objetivo deste trabalho de pesquisa foi analisar os indicadores de saúde e pobreza que influenciam o aumento de infecções pelo coronavírus tipo 2 causador da síndrome respiratória aguda grave (SARS-CoV-2) por meio de dois modelos preditivos. Uma análise foi realizada em 2.409 municípios do México em dois períodos de tempo. Para o período dois (13 de julho de 2021) as variáveis "Pneumonia", "Asma”, "Outra complicação”, "Obesidade” e "Outro caso" influenciaram a disseminação da covid-19 em 0,4024, 0,5229, 2,4246, 1,0053 e 1,4788, respectivamente. Da mesma forma, a falta de renda, moradia e alimentação foram variáveis sociais que influenciaram o número de infecções, devido ao fato de que uma "distância saudável" não poderia ser mantida. Conclui com a exigência de que o Governo aplique maiores medidas tanto de saúde como de sensibilização social para o contágio da covid-19, bem como programas sociais em locais onde existam pessoas vulneráveis devido a rendimentos, pessoas com falta de qualidade e espaços habitacionais .e pessoas com falta de acesso a alimentos.

Palavras-chave: covid-19, pandemia, pobreza, saúde, habitação.

Fecha Recepción: Julio 2021

Fecha Aceptación: Enero 2022

\section{Introducción}

En diciembre del año 2019, en la ciudad de Wuhan, provincia de Hubei, en China, inició un brote de neumonía causado por un nuevo coronavirus, el coronavirus de tipo 2 causante del síndrome respiratorio agudo severo (SARS-CoV-2). El virus se propagó de forma global y ha provocado miles de muertes y sacudido fuertemente los sistemas de salud y la economía (Ciotti et al., 2020).

Oficialmente, el 11 de marzo de 2020 la Organización Mundial de la Salud (OMS) caracterizó a la enfermedad por el coronavirus de 2019 (covid-2019) como una pandemia. Al día de hoy, luego de casi dos años, dicha pandemia aún requiere de respuestas oportunas en materia sanitaria, social y económica para sobrellevar la situación de la mejor forma posible. 


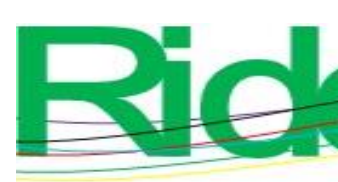

Revista Iberoamericana para la
Investigación y el Desarrollo Educativo
ISSN $2007-7467$

Por tanto, es necesario realizar investigaciones y análisis de datos para poder elaborar políticas públicas en materia de salud pública con fundamento cuantitativo. Entre estas políticas se encuentra, por ejemplo, la reasignación de presupuestos hacia temas relacionados con la salud, así como la atención de carencias sociales, de calidad de vida y espacios de vivienda y de accesibilidad a los servicios de salud.

Al inicio de la contingencia sanitaria, para evitar la propagación del virus, la Secretaría de Salud de México (11 de marzo de 2020) emitió una serie de protocolos sanitarios, lavarse las manos, mantener la distancia y el aislamiento social, entre otros. Sin embargo, estos protocolos no siempre se pudieron seguir debido a la calidad de los espacios de vivienda de algunos grupos poblacionales vulnerables.

Al analizar toda información proporcionada por fuentes oficiales surgen las siguientes interrogantes: ¿qué indicadores de salud tienen una mayor influencia para contraer la covid19? y ¿qué aspectos sociales de pobreza propician un incremento en el número de casos por covid-19? Las hipótesis que se plantean son las siguientes: 1) las enfermedades de neumonía y obesidad, entre otras, aumentan el contagio de covid-19 y 2) el contagio de covid-19 sucede cuando las personas presentan carencia por calidad y espacios de la vivienda.

Derivado de las interrogantes, el objetivo de esta investigación es analizar los indicadores de salud y de pobreza que influyen en el aumento de contagios por el SARSCoV-2 en México a través de dos modelos de regresión lineal múltiple en dos periodos de tiempo. Se realizaron dos periodos para analizar el cambio en los valores predictores de casi un año de diferencia, es decir, de junio 2020 a julio 2021.

Para poder cumplir con el objetivo de esta investigación se incluye un análisis situacional acerca de la covid-19, así como las consecuencias de este virus en el entorno social y económico, además de aspectos de la calidad de la vivienda y la salud. Posteriormente, se describe la metodología y los resultados. Por último, hay un apartado para discutir dichos resultados y otro para emitir conclusiones. 


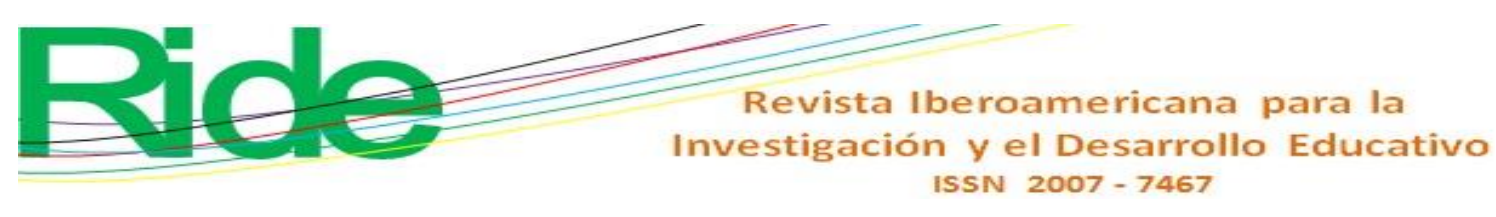

\section{Panorama general del covid-19 y situación de la pandemia en México}

La covid-19 es una enfermedad que surgió en Wuhan, una ciudad perteneciente a China. Esta enfermedad se ha propagado rápidamente a escalas internacionales. En enero de 2020, la OMS declaró el brote de covid-19 como emergencia de salud pública a nivel internacional. El primer caso declarado en la región de América Latina y el Caribe se confirmó en febrero 2020, y el brote se propagó por toda la región meses después. Acorde a datos de junio 2020, Estados Unidos y Brasil sumaban $77 \%$ del total de todos los casos y $79 \%$ de todas las muertes reportadas en la región (Organización Panamericana de la Salud [OPS], 2020).

Desde los inicios de esta emergencia sanitaria, se ha informado que la mayoría de los casos han ocurrido en personas adultas y que ciertas comorbilidades pueden asociarse con casos graves y muerte; sin embargo, también se han registrado casos en menores de un año (Ramos, 2020).

Para el día 8 de marzo del 2020, en México se habían registrado un total de siete casos en todo el país, por lo cual la Dirección General de Promoción de la Salud dio a conocer algunas medidas para evitar la propagación del covid -19, entre las cuales estaban lavarse las manos frecuentemente con agua y jabón o utilizar gel con base de alcohol a 70 \%; en caso de toser o estornudar, utilizar el estornudo de etiqueta, evitar tocarse la cara, la nariz, la boca y los ojos con las manos sucias; limpiar y desinfectar superficies y objetos de uso común en casas, oficinas, sitios cerrados y transporte, y en caso de presentar síntomas de algún padecimiento respiratorio, quedarse en casa y solo en caso de presentarse fiebre mayor a 38 ${ }^{\circ} \mathrm{C}$ y dolor de garganta o cabeza acudir al médico (Secretaría de Salud, 2020a).

Unos días más adelante, se creó la Jornada Nacional de Sana Distancia, la cual promovía el distanciamiento social, por ejemplo, en caso de tener contacto con personas fuera de la familia nuclear, estar cuando menos a 1.5 metros de distancia el uno del otro. De igual forma, las personas mayores de 60 años debían permanecer en casa en resguardo familiar. Por supuesto, se recomendaba manejar un saludo a distancia, no saludar de beso, de mano o abrazo (Secretaría de Salud, 11 de marzo de 2020). El objetivo de ambas medidas era reducir la transmisión comunitaria de covid-19 en México mediante la reducción de la tasa de contacto efectiva (Acuña, Santana y Velasco, 2020). A pesar de estas medidas, un mes después, el 8 de abril del 2020, los casos se habían incrementado en $80 \%$; oficialmente se 


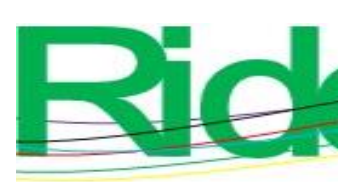

Revista Iberoamericana para la
Investigación y el Desarrollo Educativo
ISSN $2007-7467$

había reportado un total 3181 casos con 174 defunciones (Secretaría de Salud, 30 de agosto de 2021).

Para el 1 de mayo en México se habían confirmado 20739 casos y 1972 defunciones por covid-19. Consecuentemente, la Dirección General de Salud comunicó que la población debía resguardarse en casa para evitar la propagación del virus, lo cual implicaba no salir a menos que fuera estrictamente necesario. Sin embargo, no todos, no solo en México, sino también a nivel internacional, pueden permitirse cumplir con las políticas de aislamiento social.

\section{Consecuencias de covid-19 en el entorno social}

En las pandemias precedentes a la de la covid-19, las tasas de mortalidad fueron mayores en los sectores económicos más vulnerables. En efecto, las personas de estatus socioeconómico más bajo presentan peores condiciones de salud debido a que mantienen hábitos de vida menos saludables, trabajan en ocupaciones que suponen un mayor desgaste físico, están expuestos a mayores niveles de estrés y tienen un peor acceso a las prestaciones de salud (Sánchez, 2020). El covid-19 encaja dentro de esos patrones y relaciones, particularmente con los estratos socioculturales de inseguridad, vulnerabilidad y riesgo (French y Monahan, 2020).

La pandemia del covid-19 ha puesto en manifiesto las brechas socioeconómicas alrededor del mundo: los lugares más pobres sufren más contagios, los trabajadores informales que no tienen la posibilidad de quedarse en casa, debido a que su ingreso depende de salir a trabajar, se ven en la necesidad de continuar con sus actividades a pesar del riesgo sanitario. Los países con menos recursos sufren para proteger a su población (Fuentes, 2020). Mientras que algunas personas pueden quedarse en casa y realizar sus múltiples actividades desde la comodidad del hogar, otras más se ven en la necesidad de salir y exponerse a un mayor riesgo de contagio (Sánchez, 2020).

Las condiciones de pobreza y desigualdad en las que vive gran parte de la sociedad mexicana, aunadas a la fragilidad macroeconómica y a un sistema de salud fragmentado, con pocos recursos, multiplican el grado del desafío que enfrenta el país. A pesar de estas limitaciones, la magnitud de la crisis del covid-19 necesita una respuesta contundente y determinada (Fuentes, 2020). La pandemia de covid-19 está imponiendo muchos desafíos sociales. En sociedades desiguales es imperativo proteger a la población vulnerable (familias 


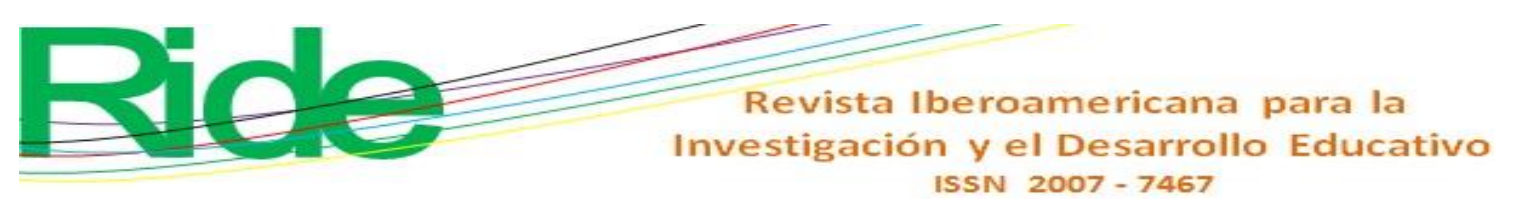

con niños y jóvenes que subsisten de la economía informal) para evitar el covid-19, debido a que esta carece de seguridad social (Vilar, Pérez, Teruel, Alonso y Pérez, 2020).

Los problemas sociales relacionados con la pandemia pueden tener un impacto considerable y duradero en las sociedades y en individuos particulares, por lo que es conveniente asegurar un apoyo de ingresos adicional para las personas socialmente desfavorecidas durante y poco después de la pandemia. De igual forma, Rudnick (2020) sugiere tomar medidas educativas para capacitar al público en general, así como a los proveedores de atención médica sobre comportamientos responsables que los protegen a ellos y a otros durante una pandemia y otros momentos difíciles.

Aún hoy en día se espera que el SARS-CoV-2 continúe infectando a millones de personas en todo el mundo, por lo que el impacto económico será grande y millones de personas serán empujadas a la pobreza. En Indonesia, por ejemplo, el crecimiento económico de $5 \%$ proyectado en 2020 pasó a estar entre $4.2 \%$ y $-4.7 \%$. Además, se pronosticaba que la tasa de pobreza aumentaría de $9.2 \%$ en septiembre de 2019 a $9.7 \%$ para fines de 2020 , lo que implicaría que 1.3 millones de personas serían empujadas a la pobreza. Por otro lado, la proyección más severa apuntaba a que la tasa de pobreza aumentaría $17.9 \%$, lo que implicaría que 23.4 millones más de personas se volverían pobres. Frente a estos datos, Indonesia necesita expandir sus programas de protección social para ayudar a los nuevos pobres, además de los pobres existentes (Suryahadi, Izzati y Suryadarma, 2020).

\section{Consecuencias de covid-19 en el entorno económico}

Esta pandemia, además de evidenciar la fragilidad de los servicios de salud, también puso de manifiesto las debilidades del modelo económico preponderante en el mundo: gran endeudamiento privado y corporativo que se refleja en las caídas continuas de todas las bolsas del mundo, máxime cuando se habla de una economía que ya se veía que estaba a punto de caer en una recesión como es la mexicana (Orellana, 2020).

En Indonesia, se estimó que la covid-19 reduciría la tasa de crecimiento económico, por lo que se otorgaron exenciones de impuestos a las empresas y trabajadores que ganan menos, así como un programa de asistencia social existente, como los programas de transferencia condicional de efectivo y asistencia alimentaria no monetaria (Suryahadi et al., 2020). En el caso de México, particularmente en la Ciudad de México, durante el primer año de pandemia se esperaba igualmente una contracción económica, lo que agravaría aún más 


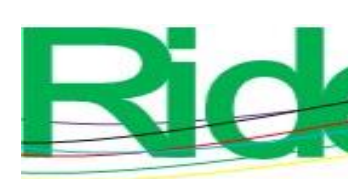

Revista Iberoamericana para la
Investigación y el Desarrollo Educativo
ISSN $2007-7467$

se puede reducir la probabilidad de transmisión. De ahí la necesidad de establecer continuamente acciones para la reducción de la transmisión de este virus en la comunidad y en los centros de atención sanitaria.

Muchos de los pacientes infectados por el SARS-CoV-2 presentan las condiciones de neumonía, asma y obesidad; resulta significativo analizar de manera general el cómo estas condiciones de salud colocan a la población con dichas enfermedades en el sector vulnerable.

\title{
Neumonía
}

La neumonía por covid-19 se puede detectar a partir anomalías en las imágenes de tomografía computarizada del tórax, incluso en pacientes asintomáticos, pues las lesiones pueden evolucionar rápidamente a un patrón de predominio o consolidación de opacidad de vidrio esmerilado difuso dentro de una a tres semanas después del inicio de los síntomas, alcanzando un máximo alrededor de dos semanas después del inicio. Asimismo, la vejez, el sexo masculino, las comorbilidades subyacentes y el deterioro radiográfico progresivo pueden ser factores de riesgo para el pronóstico en pacientes con neumonía por covid-19 (Shi et al., 2020).

\begin{abstract}
Asma
Cuando se trata y se monitorea de cerca, el asma no es potencialmente mortal, sin embargo, si alguien que lo padece tiene un ataque de asma y no tiene acceso a un inhalador o ventilador puede ser fatal, por lo que los profesionales médicos advierten a las personas con asma que tengan cuidado. Además, debido a que no ha habido información que distinga los ataques de asma de los síntomas comunes de covid-19, los síntomas relacionados con el virus pueden confundirse con un ataque de asma de rutina y disuadir a las personas de buscar atención médica (Cione et al., 2020).
\end{abstract}

\section{Obesidad}

La respuesta de México al covid-19 se ve obstaculizada por un doble desafío sinérgico: las enfermedades no transmisibles como la obesidad, la diabetes y la hipertensión, así como la desigualdad social; además de que estas enfermedades aumentan la probabilidad de una enfermedad grave y la muerte (Gutierrez y Bertozzi, 2020). 

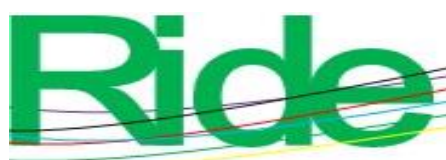

Revista Iberoamericana para la Investigación y el Desarrollo Educativo ISSN 2007 - 7467

medición de la pobreza a escala municipal en México. Así, se obtuvieron 2409 registros de datos de pobreza por municipio de cada entidad federativa.

\section{Análisis de datos}

Se realizó una base de datos con aspectos de salud en cuanto a pacientes con covid19. Como parte de este proceso, se realizaron las tablas de datos correspondientes en cuanto aspectos de salud y geográficos. Posteriormente, se realizó una unión de datos por municipio y entidad federativa y se añadieron los datos de pobreza. Asimismo, se realizaron correlaciones de datos y se seleccionaron las variables que se presentan en la figura 2 y se describen en la tabla 1, por ser las de más alta correlación con el contagio por covid-19.

Figura 2. Variables de estudio utilizadas para el modelo predictivo de contagio por covid-

19

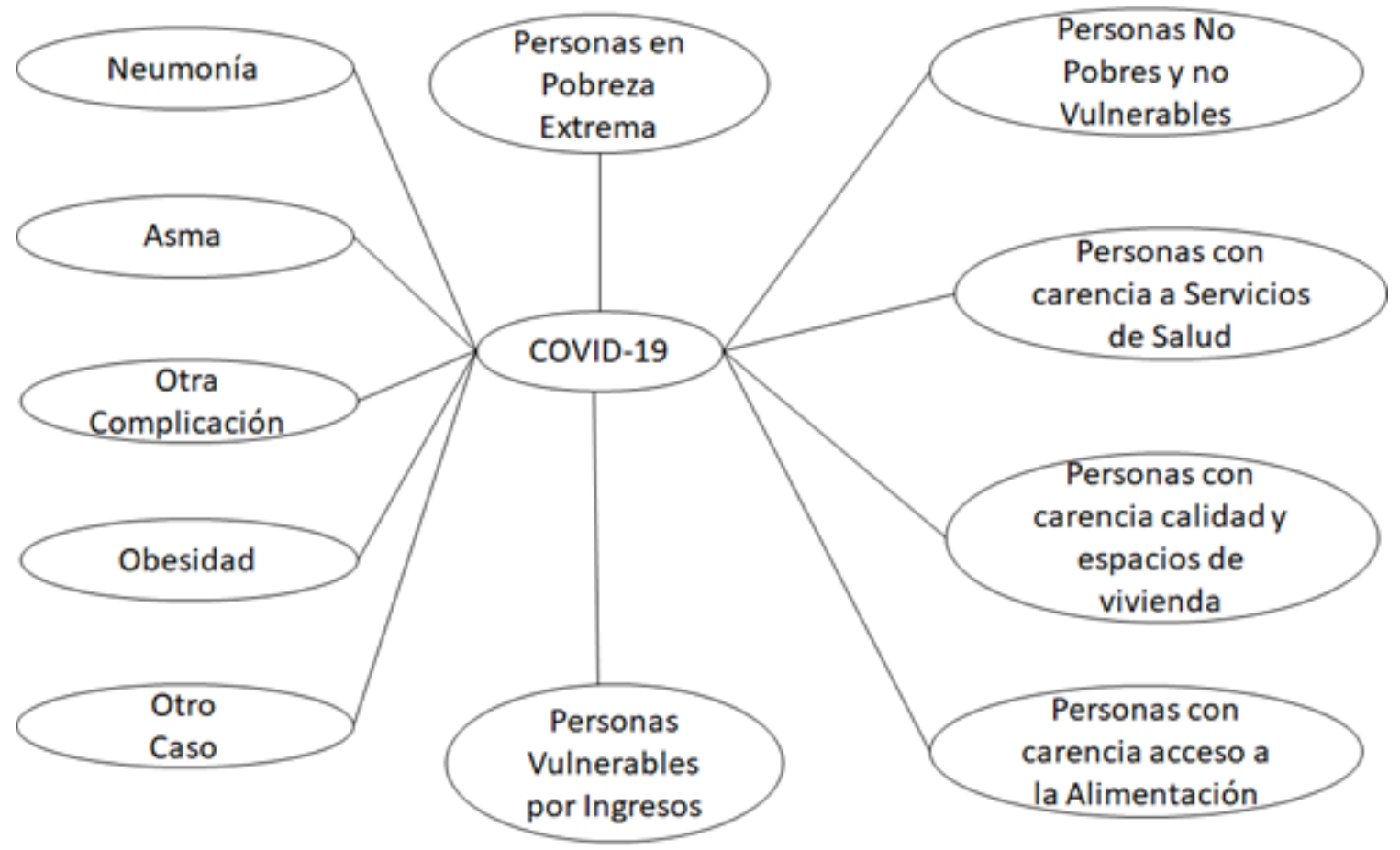

Fuente: Elaboración propia 


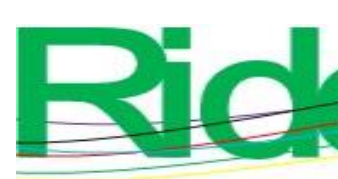

Revista Iberoamericana para la Investigación y el Desarrollo Educativo ISSN 2007 - 7467

Tabla 1. Descripción de variables utilizadas en el modelo predictivo de contagio del covid-

19

\begin{tabular}{|c|c|}
\hline Variable & Descripción \\
\hline Covid-19 (Y) & $\begin{array}{l}\text { Número de personas con covid-19 con resultado Positivo SARS- } \\
\text { CoV-2, agrupados por municipio y entidad de residencia }\end{array}$ \\
\hline Neumonía $\left(\mathrm{X}_{1}\right)$ & Paciente con diagnóstico de neumonía \\
\hline $\operatorname{Asma}\left(\mathrm{X}_{2}\right)$ & Paciente con diagnóstico de asma \\
\hline Otra complicación $\left(\mathrm{X}_{3}\right)$ & $\begin{array}{l}\text { Paciente con diagnóstico de otras enfermedades y con exclusión de } \\
\text { neumonía, asma. Enfermedad pulmonar obstructiva crónica } \\
\text { (EPOC), diabetes, inmunosupresión, hipertensión, cardiovascular, } \\
\text { obesidad, insuficiencia renal crónica y hábito de tabaquismo }\end{array}$ \\
\hline Obesidad $\left(\mathrm{X}_{4}\right)$ & Paciente con diagnóstico de obesidad \\
\hline Otro caso $\left(\mathrm{X}_{5}\right)$ & $\begin{array}{l}\text { Identifica si el paciente tuvo contacto con algún otro caso } \\
\text { diagnosticado con SARS-CoV-2 }\end{array}$ \\
\hline $\begin{array}{l}\text { Personas en pobreza } \\
\text { extrema }\left(\mathrm{X}_{6}\right)\end{array}$ & $\begin{array}{l}\text { Indicador de pobreza acerca de personas en situación de pobreza } \\
\text { extrema }\end{array}$ \\
\hline $\begin{array}{l}\text { Personas vulnerables por } \\
\text { ingresos }\left(\mathrm{X}_{7}\right)\end{array}$ & $\begin{array}{l}\text { Indicador de pobreza sobre personas que no presentan carencias } \\
\text { sociales y su ingreso es menor o igual a la línea de bienestar } \\
\text { (Coneval, 2014) }\end{array}$ \\
\hline $\begin{array}{l}\text { Personas no pobres y no } \\
\text { vulnerables }\left(\mathrm{X}_{8}\right)\end{array}$ & $\begin{array}{l}\text { Indicador de pobrezas sobre personas cuyo ingreso es superior a la } \\
\text { línea de bienestar y que no tiene carencia social alguna (Coneval, } \\
2014 \text { ) }\end{array}$ \\
\hline $\begin{array}{l}\text { Personas con carencia de } \\
\text { servicios de salud }\left(\mathrm{X}_{9}\right)\end{array}$ & $\begin{array}{l}\text { Se considera que una persona se encuentra en situación de carencia } \\
\text { por acceso a los servicios de salud cuando no cuente con adscripción } \\
\text { o derecho a recibir servicios médicos de alguna institución que } \\
\text { preste servicios médicos, incluyendo al Seguro Popular, a las } \\
\text { instituciones de seguridad social (Instituto Mexicano del Seguro } \\
\text { Social [IMSS], Instituto de Seguridad y Servicios Sociales para los } \\
\text { Trabajadores del Estado [Issste], Pemex, Ejército o Marina) o los } \\
\text { servicios médicos privados (Coneval, 2014) }\end{array}$ \\
\hline
\end{tabular}




\begin{tabular}{|l|l|}
$\begin{array}{c}\text { Revista Iberoamericana para la } \\
\text { Investigación y el Desarrollo Educativo } \\
\text { ISSN } 2007-7467\end{array}$ \\
$\begin{array}{l}\text { Personas con carencia en } \\
\text { calidad y espacios de } \\
\text { vivienda }\left(\mathrm{X}_{10}\right)\end{array}$ & $\begin{array}{l}\text { Son las personas que residen en viviendas que presentan, al menos, } \\
\text { una de las siguientes características: 1) el material de los pisos es de } \\
\text { tierra, 2) el material del techo es de lámina de cartón o desechos, 3) } \\
\text { el material de los muros es de embarro o bajareque, de carrizo, } \\
\text { bambú o palma, de lámina de cartón, metálica o asbesto, o material } \\
\text { de desecho y 4) la razón de personas por cuarto (hacinamiento) es } \\
\text { mayor que 2.5 (Coneval, 2014) }\end{array}$ \\
\hline $\begin{array}{l}\text { Personas con carencia de } \\
\text { acceso a la alimentación }\end{array}$ & $\begin{array}{l}\text { Es la población que vive en hogares que presentan un grado de } \\
\text { inseguridad alimentaria moderado o severo (Coneval, 2014) }\end{array}$ \\
\hline
\end{tabular}

Fuente: Elaboración propia con base en el Coneval (2014) y la Dirección General de

Epidemiología (Secretaría de Salud, 30 de agosto de 2021)

\section{Modelo}

Se realizaron dos modelos de regresión lineal múltiple por el método de mínimos cuadrados ordinarios tanto para el periodo uno como el dos con el software estadístico Gretl 2021. Esto con el fin de medir el efecto de las variables de salud y de pobreza que se presentan en la tabla 1 sobre los casos de contagio de covid-19. Como variable dependiente se tiene "Número de casos confirmados de covid-19 por municipio de México". Como variables independientes se tiene "Neumonía”, “Asma”, "Otra complicación”, "Obesidad”, "Otro caso", "Personas en pobreza extrema", "Personas vulnerables por ingresos", "Personas no pobres y no vulnerables", "Personas con carencia de servicios de salud", "Personas con carencia en calidad y espacios de vivienda" y "Personas con carencia de acceso a la alimentación", lo cual queda representado para los modelos en los diferentes periodos en la ecuación 1.

$$
Y=\beta_{0}+\beta_{1} X_{1}+\cdots+\beta_{11} X_{11}+u
$$



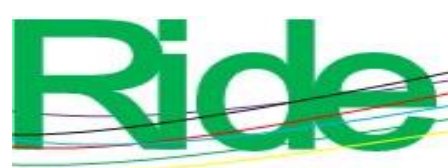

Revista Iberoamericana para la Investigación y el Desarrollo Educativo ISSN $2007-7467$

\section{Escenario con variable: "Personas con carencia en calidad y espacios de vivienda" $\mathrm{y}$ "Contagios por covid-19"}

Se realizó un escenario con la variable independiente de pobreza "Escenario covid19” y "Acciones para disminuir el número de personas con carencia en calidad y espacios de vivienda". Para ello, se realizó una estimación de la variable con los coeficientes estimadores del modelo del periodo dos, comprendido entre el 1 de enero de 2020 al 13 de julio de 2021, y se consideraron los valores promedio de las variables. El escenario fue realizado con la ecuación 2.

$$
\begin{gathered}
\hat{Y}=-\beta_{0}+\beta_{1} \bar{X}_{1}+\beta_{2} \bar{X}_{2}+\beta_{3} \bar{X}_{3}+\beta_{4} \bar{X}_{4}+\beta_{5} \bar{X}_{5}+\beta_{6} \bar{X}_{6}+\beta_{7} \bar{X}_{7}- \\
\beta_{8} \bar{X}_{8}+\beta_{9} \bar{X}_{9}+\beta_{10} \bar{X}_{10}+\beta_{11} \bar{X}_{11}
\end{gathered}
$$

\section{Resultados}

En la figura 3 se presentan los casos de contagio por covid-19 durante el periodo entre enero del 2020 y junio del 2021. De igual forma, en la figura 4 se tienen los resultados de las variables de salud del covid-19. Allí se aprecia claramente que algunas variables han tenido un crecimiento, pero tienen un comportamiento similar en ambos periodos; las más representativas: "Neumonía”, "Diabetes", "Hipertensión”, "Obesidad” y "Tabaquismo”. Por otro lado, la variable "Otro caso", la cual es la más elevada, se refiere a haber estado en contagio con personas que tienen covid-19. En la figura 5 se tiene el número de personas relacionadas con pobreza, y se percibe la desigualdad económica, pues una gran cantidad de personas no son pobres y otra cantidad similar tiene la carencia de acceso a la alimentación. 
Figura 3. Contagios de covid-19 por mes

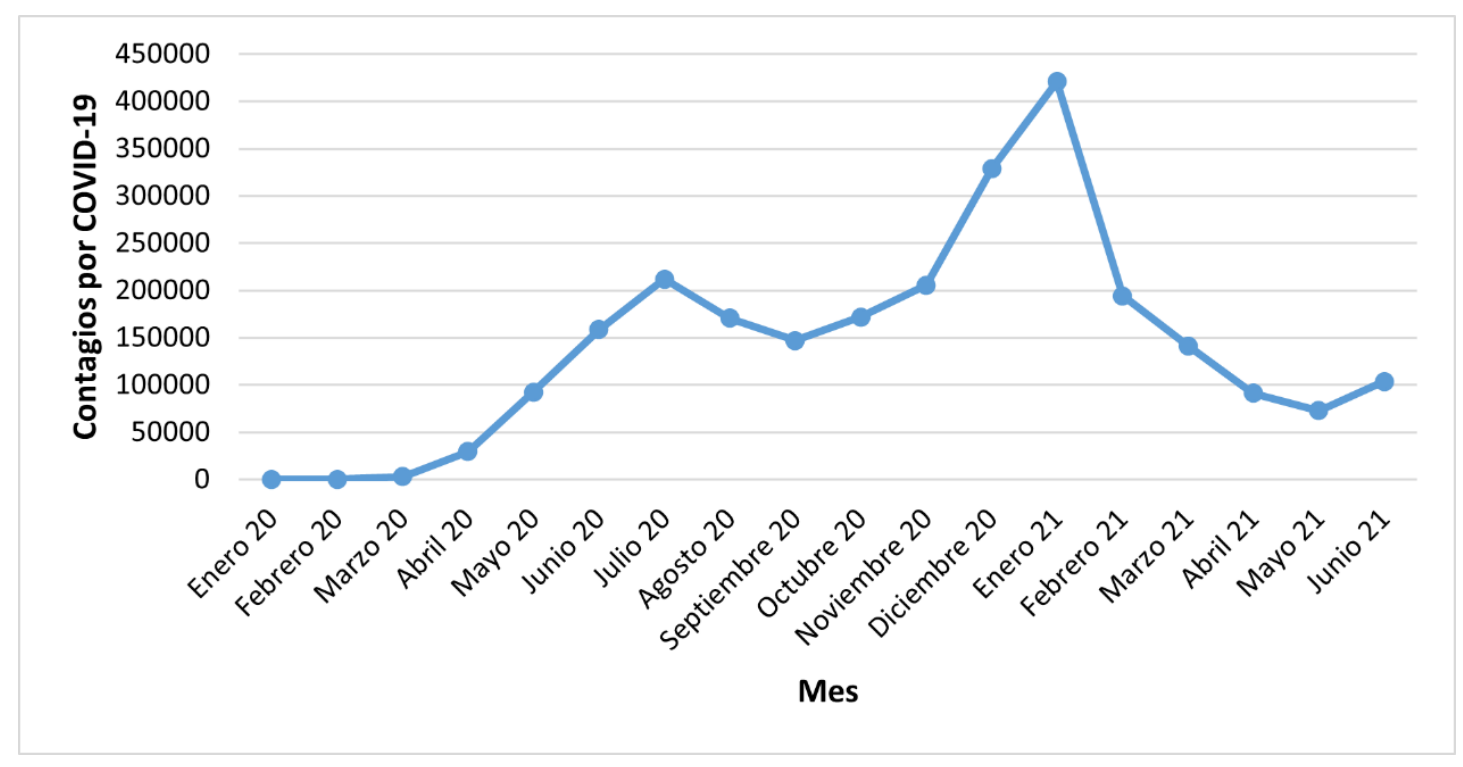

Fuente. Elaboración propia a partir de datos abiertos de covid-19 de la Secretaría de Salud en México

Figura 4. Número de casos de enfermedades de las variables de salud

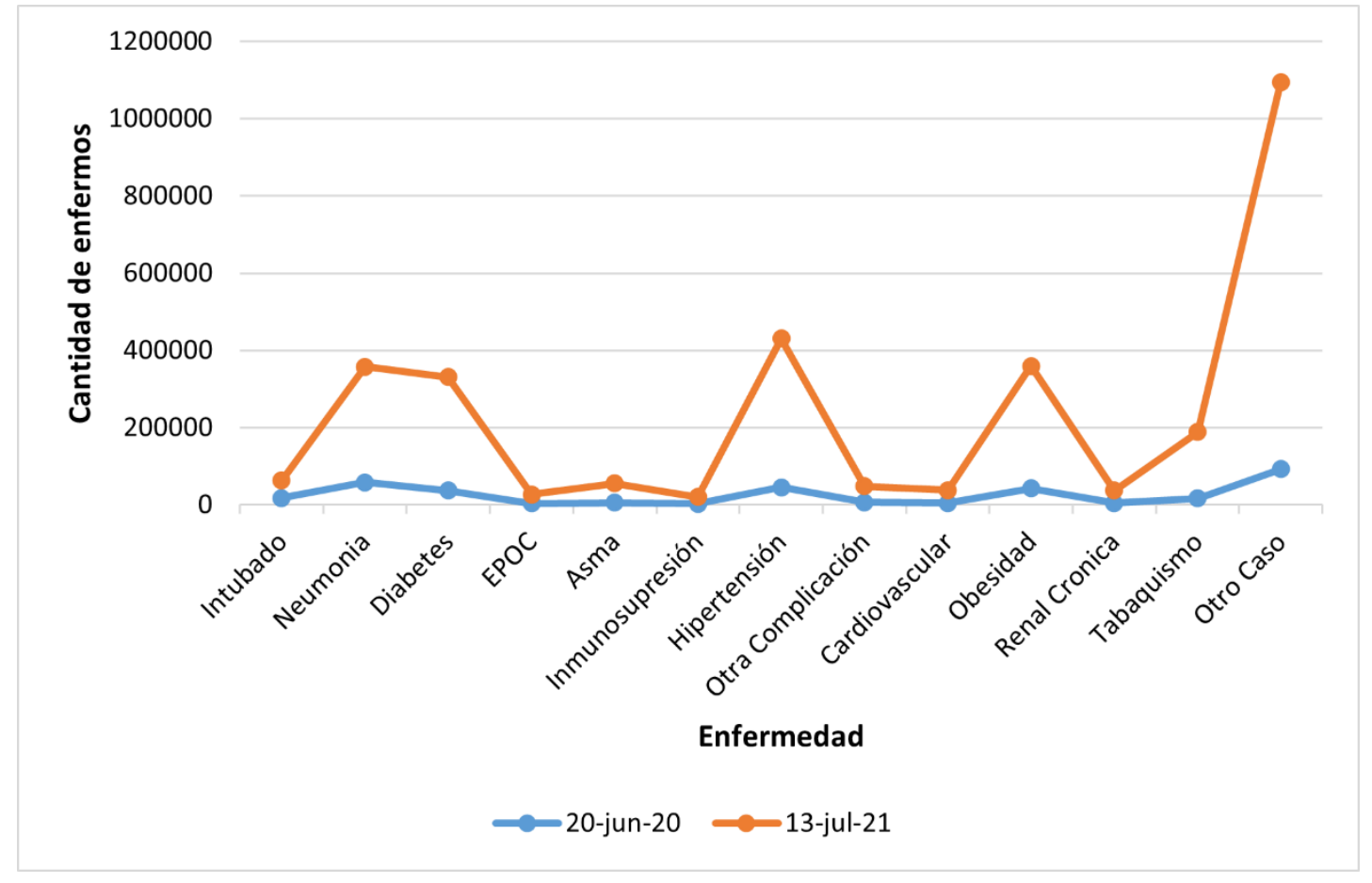

Fuente. Elaboración propia a partir de datos abiertos de covid-19 de la Secretaría de Salud en México 

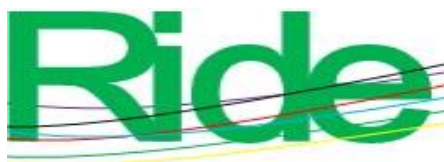

Revista Iberoamericana para la Investigación y el Desarrollo Educativo

ISSN 2007 - 7467

Figura 5. Número de personas relacionados con pobreza

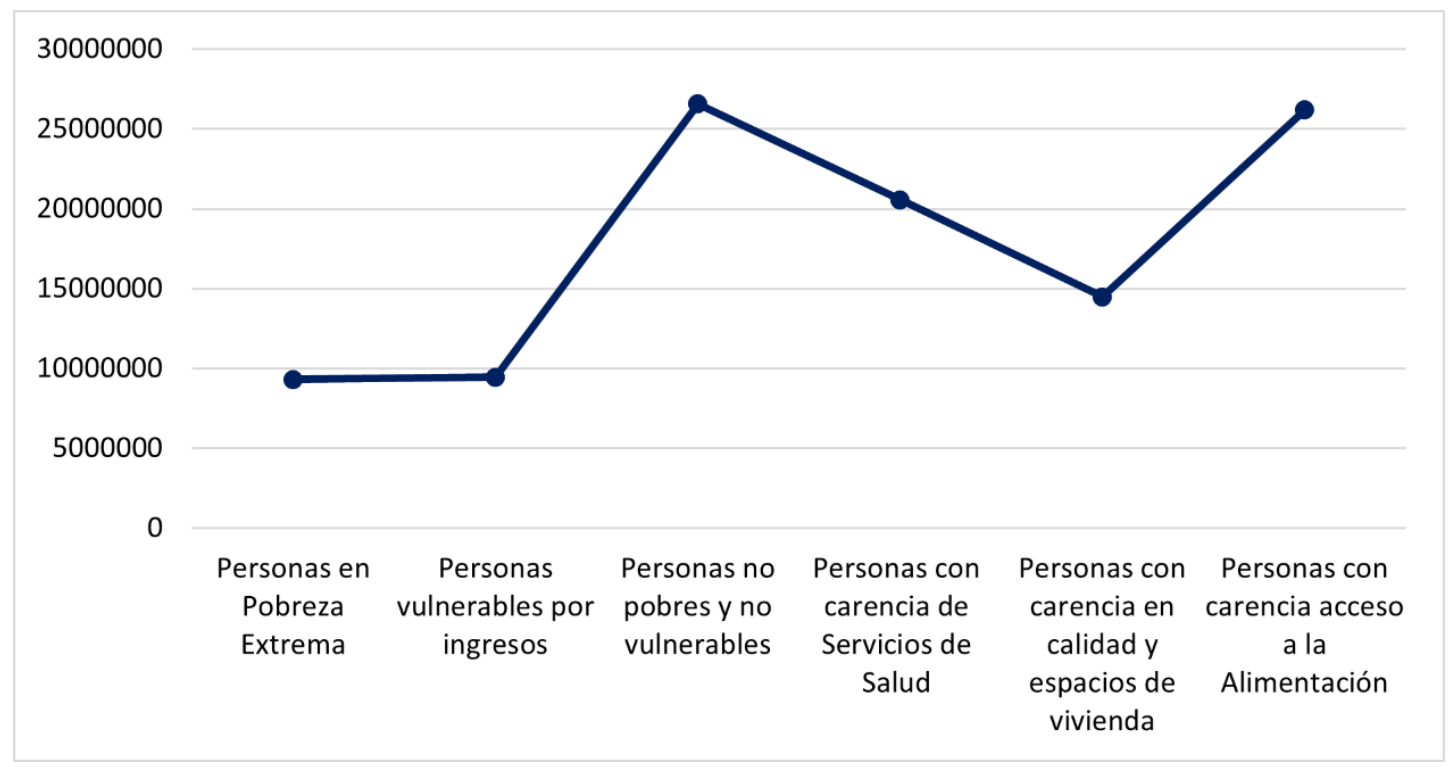

Fuente: Coneval (2015)

\section{Modelo empírico}

Para probar la hipótesis de investigación que condiciona a un aumento del covid-19 de la población de los municipios de México, se presentan en la tabla 2 los resultados de los modelos que incluyen los coeficientes estimados y estadísticos de decisión, así como la prueba de $F$ como validez de los modelos. 


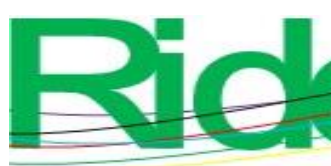

\section{Revista Iberoamericana para la Investigación y el Desarrollo Educativo ISSN 2007 - 7467}

Tabla 2. Estimaciones de los parámetros de regresión del modelo predictivo de contagios por covid-19

\begin{tabular}{|c|c|c|c|c|c|c|}
\hline \multicolumn{7}{|c|}{ Variable dependiente: "Contagio por covid-19" } \\
\hline & \multicolumn{3}{|c|}{$\begin{array}{c}\text { Periodo uno: } \\
1-01-2020 \text { a 20-06-2020 }\end{array}$} & \multicolumn{3}{|c|}{$\begin{array}{c}\text { Periodo dos: } \\
1-01-2020 \text { a 13-07-2021 }\end{array}$} \\
\hline Parámetro & Coef & Valor $\mathrm{p}$ & & Coef & Valor $\mathrm{p}$ & \\
\hline (Constante) & -3.363 & 0.007 & $* *$ & -21.6885 & 0.1218 & \\
\hline Neumonía & 0.9358 & $<0.0001$ & $* * *$ & 0.4024 & $<0.0001$ & $* * *$ \\
\hline Asma & 0.3008 & 0.093 & $*$ & 0.5229 & 0.301 & \\
\hline Otra complicación & 1.9973 & $<0.0001$ & $* * *$ & 2.4246 & $<0.0001$ & $* * *$ \\
\hline Obesidad & 1.0427 & $<0.0001$ & $* * *$ & 1.0053 & $<0.0001$ & $* * *$ \\
\hline Otro caso & 0.1481 & $<0.0001$ & $* * *$ & 1.4788 & $<0.0001$ & $* * *$ \\
\hline $\begin{array}{l}\text { Personas en pobreza } \\
\text { extrema }\end{array}$ & -0.002 & $<0.0001$ & $* * *$ & -0.0192 & $<0.0001$ & $* * *$ \\
\hline $\begin{array}{l}\text { Personas vulnerables } \\
\text { por ingresos }\end{array}$ & -0.0007 & $<0.0001$ & $* * *$ & 0.0072 & 0.0053 & $* * *$ \\
\hline $\begin{array}{l}\text { Personas no pobres } \mathrm{y} \\
\text { no vulnerables }\end{array}$ & -0.001 & $<0.0001$ & $* * *$ & 0.0111 & $<0.0001$ & $* * *$ \\
\hline $\begin{array}{l}\text { Personas con carencia } \\
\text { de servicios de salud }\end{array}$ & 0.0003 & 0.037 & $* *$ & -0.0147 & $<0.0001$ & $* * *$ \\
\hline $\begin{array}{l}\text { Personas con carencia } \\
\text { en calidad y espacios } \\
\text { de vivienda }\end{array}$ & 0.0024 & $<0.0001$ & $* * *$ & 0.0151 & $<0.0001$ & $* * *$ \\
\hline \multirow[t]{2}{*}{$\begin{array}{l}\text { Personas con carencia } \\
\text { en acceso a la } \\
\text { alimentación }\end{array}$} & -0.0004 & $<0.0001$ & $* * *$ & 0.0112 & $<0.0001$ & $* * *$ \\
\hline & \multicolumn{3}{|c|}{$\begin{array}{c}F=11575.04 \text { Valor } p(\text { de } F) \\
0.0000\end{array}$} & \multicolumn{3}{|c|}{$\begin{array}{c}F=18835.94 \text { Valor } p(\text { de } F) \\
0.0000\end{array}$} \\
\hline
\end{tabular}

Fuente: Elaboración propia a partir de los resultados de los modelos

Los coeficientes de la relación funcional de número de casos del covid-19 con las variables de salud ("Neumonía”, “Asma”, “Otra complicación”, “Obesidad” y “Otro caso”), presentadas en la ecuación 1 resultaron con signos positivos para ambos periodos de tiempo 


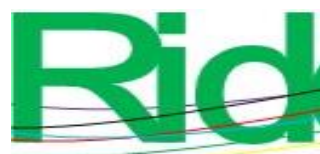

$\sum$

y fueron estadísticamente significativos según el estadístico $t$, ya que resultaron significativamente diferentes de cero a un nivel de $5 \%$.

\section{Escenario con variables de pobreza}

Se presenta un escenario: "Escenario covid-19 y carencia de calidad de vivienda". Dicho escenario mide el comportamiento del covid-19 con cambios en acciones de $10 \%$ y $20 \%$, enfocados a disminuir el número de personas con carencia en calidad y espacios de vivienda. Sin duda el Gobierno mexicano debe buscar las acciones convenientes para poder disminuir esa carencia. Dichas acciones pueden ser otorgar facilidades para hacer espacios de sanitización, así como suministrar el servicio de agua potable con una mayor frecuencia, o bien otorgar material para hacer cuartos y evitar el hacinamiento, de tal forma que se pueda realizar la "sana distancia" tanto en espacios para dormir como de convivencia. Esto se muestra en la figura 6.

Figura 6. Escenario de personas con carencia en calidad y espacios de vivienda y covid-19

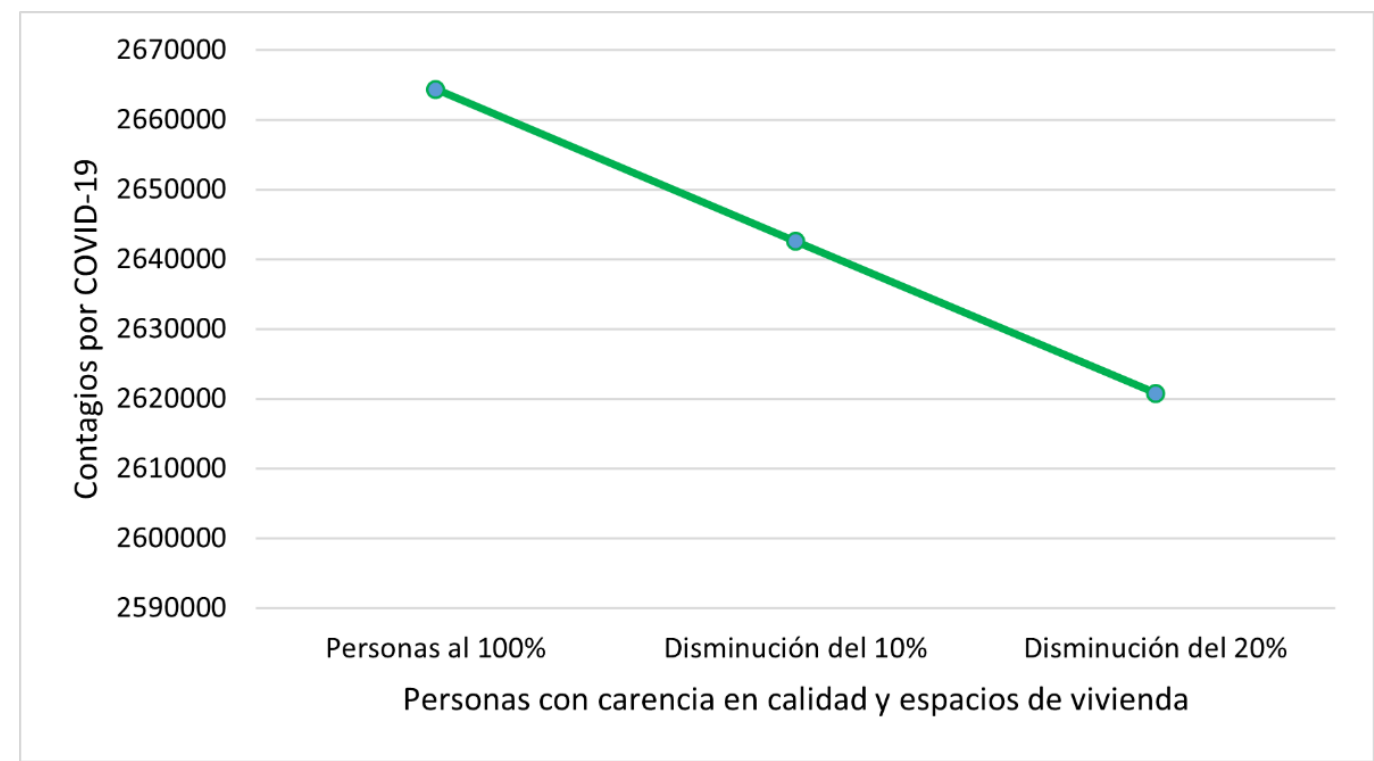

Fuente: Elaboración propia 


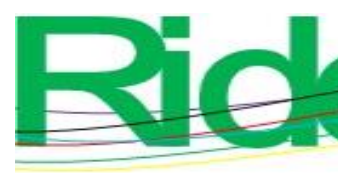

Revista Iberoamericana para la
Investigación y el Desarrollo Educativo
ISSN $2007-7467$

Asimismo, respecto a la variable "Personas con carencia en calidad y espacios de vivienda", se presentan para ambos periodos signos positivos, el más reciente de 0.0151 ; en la tabla 2 se aprecia que dicho predictor creció, lo cual indica que pueden adquirir el covid19 por tener esta carencia. Esto resulta importante analizar pues existe población con estas carencias, las cuales no pueden seguir las medidas de sanitización por no tener calidad y espacios de la vivienda, es decir, no se pueden lavar las manos tantas veces como se requiera, además de no tener acceso a los servicios de agua potable y vivir en hacinamiento, por lo cual no pueden tampoco mantener la sana distancia con los familiares que salen diariamente a trabajar.

Para la variable "Personas con carencia en acceso a la alimentación”, el coeficiente para el periodo dos resultó positivo (0.01129); este coeficiente para el periodo uno era negativo (-0.0004), lo cual indica que el covid-19 se puede contagiar por tener esta carencia de alimentación.

Esta investigación complementa el trabajo de otros autores. Por ejemplo, Fuentes (2020) dice que los barrios más pobres en su país sufren más contagios. Sin embargo, aquí en México se está aportando el tipo de carencias (personas vulnerables por ingresos, calidad y espacios de vivienda y acceso a la alimentación), que son las que tienen un impacto para aumentar el número de contagios por covid-19.

Por otro lado, Sánchez (2020) también comentó sobre las personas que tienen que continuar con su vida diaria, y aunque usen cubrebocas y guantes, no cambia el riesgo de contagiarse de la covid-19. Esto tiene relación con la variable "Otro caso", pues son las personas que han estado en contacto con personas contagiadas de covid-19, tal es el caso del personal de salud, que se han visto contagiados en zonas donde no existe la carencia de calidad y espacios de vivienda y, en consecuencia, se quedaron sin servicios de salud, algunos de manera definitiva (fallecimiento) y otros de forma temporal (cuando los médicos de contagiaron y guardaron la cuarentena). De ahí la urgencia de formular políticas para que el Gobierno proporcione apoyo a las comunidades vulnerables por estas carencias, según Vilar et al. (2020).

Es importante también capacitar a las comunidades con las carencias de calidad y espacios de la vivienda para que tomen conciencia del covid-19 (Rudnick, 2020). Esto también está en concordancia con los resultados, pues el Gobierno mexicano tendrá que invertir en acciones tanto para disminuir las carencias de calidad y espacios de vivienda como de alimentación. Para ello, en la sección de resultados se presenta un escenario que indica el 


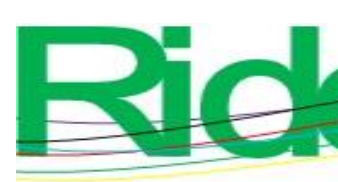

Revista Iberoamericana para la Investigación y el Desarrollo Educativo ISSN $2007-7467$

porcentaje de acciones que el Gobierno tiene que considerar para disminuir la carencia social de calidad y espacios de vivienda y reducir de esta forma los casos de contagio por covid-19.

Por otro lado, Orellana (2020) planteó que en México se espera una recesión económica debido a la pandemia. De igual forma, el Banco Mundial (2020) proyectó un aumento en las tasas de pobreza y desigualdad. Es importante considerar que en México se tienen 20.5 millones de personas con carencia de acceso a los servicios de salud, por lo que urge que el Gobierno tome las medidas económicas necesarias.

Las limitaciones de este trabajo son la carencia de un seguimiento por paciente, debido a que no se cuentan con estadísticas de acceso abierto que incluyan ese dato.

\section{Conclusiones}

El objetivo se cumplió y se analizaron los indicadores de salud y pobreza que influyen en el aumento de contagios por el covid-19 y, derivado de los resultados, se concluye que es necesario que el Gobierno aplique mayores medidas tanto de salud como de conciencia social al contagio del covid-19, así como de programas sociales en los municipios donde exista: 1) personas vulnerables por ingresos, 2) personas con carencia en calidad y espacios de vivienda y 3 ) personas con carencia en acceso a la alimentación, esto aplica tanto a zonas urbanas y rurales. Pues las personas con estas carencias no pueden seguir las medidas sanitarias como lavarse las manos con frecuencia, pues no tienen los espacios para hacerlo, además de que algunas personas viven en hacinamiento y salen diario a trabajar y regresan a contagiar a la familia. De igual forma, ampliar los servicios de salud para prevenir enfermedades como neumonía, asma, obesidad y otras complicaciones para disminuir el contagio del covid-19. Es importante considerar el escenario que se presenta en la sección de resultados en donde se muestra que, con acciones de por lo menos $10 \%$ para disminuir las carencias de calidad y espacios de vivienda, se puede disminuir el número de casos de contagios por covid-19.

Se sugiere incrementar las medidas de concientización en las personas, pues, aunque se transmite en los medios masivos como la televisión, no todas las personas tienen acceso, sobre todo en las comunidades rurales, en donde se tiene carencias y no ven la televisión, por falta de tiempo o por no tener energía eléctrica, o por no tener señal de televisión digital, o por vivir en hacinamiento y no tener espacios para ver la televisión (noticias). Urge que el Gobierno mexicano tome medidas sobre las carencias sociales para evitar el aumento o rebrote de covid-19 en zonas donde existe un mayor índice de pobreza. 

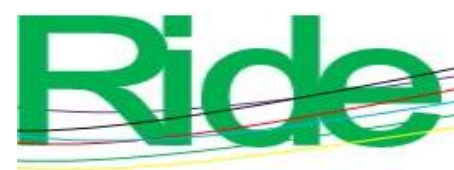

Revista Iberoamericana para la Investigación y el Desarrollo Educativo

ISSN $2007-7467$

Asimismo, las fortalezas de investigación son la presentación del escenario relacionado con la carencia de calidad y espacios de vivienda para ayudar a que el Gobierno realice las gestiones necesarias para reducir dicha carencia de calidad y tener un menor contagio y propagación del virus.

Por otro lado, la debilidad que se presenta en esta investigación es la falta de datos relacionados con: 1) aspectos económicos por cada persona que fue contagiada, 2) contagio por segunda ocasión rebrote y 3) días de recuperarse de cada paciente, pues se considera que estos serían indicadores adecuados para hacer otros modelos con aspectos de salud y pobreza.

\section{Futuras líneas de investigación}

Los resultados que fueron encontrados contribuyen para estudiar en profundidad la interacción entre las personas y la movilidad en las viviendas desde el punto de vista calidad y espacio de la vivienda. De igual forma, contribuyen para realizar un análisis de las enfermedades de neumonía, asma y obesidad en la población para evitar los contagios del covid-19.

\section{Agradecimientos}

Al Instituto Politécnico Nacional, proyecto SIP 20210776 "El impacto del covid-19 en las comunidades rurales con pobreza". 


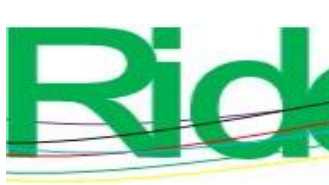

Revista Iberoamericana para la Investigación y el Desarrollo Educativo ISSN 2007 - 7467

\section{Referencias}

Acuña, M. A., Santana, M. and Velasco, J. X. (2020). Modeling behavioral change and COVID-19 containment in Mexico: A trade-off between lockdown and compliance. $\begin{array}{llll}\text { Mathematical } & \text { Biosciences, } & 325 . & \text { Retrieved }\end{array}$ https://doi.org/10.1016/j.mbs.2020.108370.

Banco Mundial. (2020). Estudio sobre el gasto social de la Ciudad de México. Banco Mundial. Recuperado de https://documentos.bancomundial.org/es/publication/documentsreports/documentdetail/192261592559856959/estudio-sobre-el-gasto-social-de-laciudad-de-méxico.

Bienvenido, D. (2021). Do unemployment benefits and economic aids to pay electricity bills remove the energy poverty risk of Spanish family units during lockdown? A study of covid-19-induced lockdown. Energy Policy, 150. Retrieved from https://doi.org/10.1016/j.enpol.2020.112117.

Castresana, A. (2020). La higiene como base de la prevención de enfermedades. En Vázquez, A. y Cambero, S. (coords.), Reflexiones desconfinadas para la era poscovid-19 (1. ${ }^{\mathrm{a}}$ ed.) (pp. 45-54). Badajoz, España: AntropiQa 2.0. Recuperado de https://dialnet.unirioja.es/servlet/libro?codigo=768210.

Cione, C., Castaneda, E., Ferdinando, A., Prince, J., Jackson, D., Vetter, E. and McCarthy, S. (2020). COVID-19 Susceptibility Among Latin People in El Paso, TX. SSRN Electronic Journal, 1-31. Retrieved from https://doi.org/10.2139/ssrn.3608396.

Ciotti, M., Angeletti, S., Minieri, M., Giovannetti, M., Benvenuto, D., Pascarella, S., Sagnelli, C., Bianchi, M., Bernardini, S. and Ciccozzi, M. (2020). COVID-19 Outbreak: An Overview. Chemotherapy, 64(5-6), 215-223. Retrieved from https://doi.org/10.1159/000507423.

Consejo Nacional de Evaluación de la Política de Desarrollo Social [Coneval]. (2010). Medición de la pobreza. Calidad y espacios en la vivienda. Recuperado de https://www.coneval.org.mx/Medicion/Paginas/Medición/Calidad-y-espacios-en-lavivienda.aspx.

Consejo Nacional de Evaluación de la Política de Desarrollo Social [Coneval]. (2014). Metodología para la medición multidimensional de la pobreza en México (3. ${ }^{a}$ ed.). Ciudad de México, México: Consejo Nacional de Evaluación de la Política de 


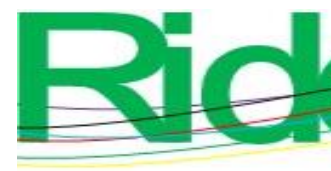

Desarrollo

Social.

Recuperado

de https://www.coneval.org.mx/InformesPublicaciones/InformesPublicaciones/Docum ents/Metodologia-medicion-multidimensional-3er-edicion.pdf.

Consejo Nacional de Evaluación de la Política de Desarrollo Social [Coneval]. (2015). Consulta dinámica de resultados de pobreza a nivel municipio 2010 y 2015. Recuperado de https://www.coneval.org.mx/Medicion/Paginas/consulta_pobreza_municipal.aspx.

French, M. and Monahan, T. (2020). Disease Surveillance: How Might Surveillance Studies Address COVID-19? Surveillance and Society, 18(1), 1-11. Retrieved from https://doi.org/10.24908/ss.v18i1.13985.

Fuentes, R. (2020). México y el coronavirus: pasividad gubernamental en una sociedad desigual. Recuperado de https://doi.org/10.33960/ac_16.2020.

Gutierrez, J. P. and Bertozzi, S. M. (2020). Non-communicable diseases and inequalities increase risk of death among COVID-19 patients in Mexico. PLoS ONE, 15(10), 111. Retrieved from https://doi.org/10.1371/journal.pone.0240394.

López, M., Quesada, J. F. y López, Ó. (2019). Calidad de vida relacionada con la salud y determinantes sociodemográficos en adultos de Cuenca, Ecuador. Revista Economía y Política, (29), 67-85. Recuperado de https://doi.org/10.25097/rep.n29.2019.04.

Orellana, J. E. (2020). El coronavirus 19 (covid-19) en México. Revista Chilena de Salud Pública, 24(1), 72-73. Recuperado de https://doi.org/10.5354/07195281.2020 .57588 .

Ramos, C. (2020). Covid-19: la nueva enfermedad causada por un coronavirus. Salud Pública de México, 62(2), 225-227. Recuperado de https://doi.org/10.21149/11276.

Rudnick, A. (2020). On pandemics and loneliness. Societies, 10(42), 1-6.

Organización de las Naciones Unidas para la Alimentación y la Agricultura [FAO]-Comisión Económica para América Latina y el Caribe [Cepal]. (2021). Sistemas alimentarios y COVID-19 en América Latina y el Caribe. Cómo fortalecer los sistemas alimentarios urbanos. Santiago, Chile: Organización de las Naciones Unidas para la Alimentación y la Agricultura- Comisión Económica para América Latina y el Caribe. Recuperado de https://doi.org/10.4060/cb5312es.

Organización Mundial de la Salud [OMS]. (11 de marzo de 2020). Alocución de apertura del Director General de la OMS en la rueda de prensa sobre la covid-19 celebrada el 11 de marzo de 2020. Recuperado de https://www.who.int/es/director- 


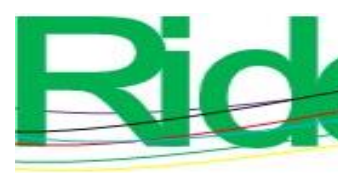

\section{Revista Iberoamericana para la Investigación y el Desarrollo Educativo ISSN $2007-7467$}

general/speeches/detail/who-director-general-s-opening-remarks-at-the-mediabriefing-on-covid-19---11-march-2020.

Organización Panamericana de la Salud [OPS]. (2020). Respuesta de la Organización Panamericana de la Salud a la COVID-19 en la Región de las Américas, del 17 de enero al 31 de mayo del 2020. Organización Panamericana de la Salud.

Sánchez, M. V. (2020). La desigualdad social perjudica seriamente la salud: el coronavirus sí entiende de clases sociales. En Vázquez, A. y Cambero, S. (coords.), Reflexiones desconfinadas para la era poscovid-19 (1. ${ }^{\mathrm{a}}$ ed.) (pp. 74-87). Badajoz, España: $\begin{array}{llll}\text { AntropiQa } & 2.0 \text { Recuperado }\end{array}$ https://dialnet.unirioja.es/servlet/libro?codigo $=768210$.

Santa, R. (2020). La importancia de la vivienda para el cuidado de la salud en el Perú, en el marco de la pandemia covid-19. (Trabajo de investigación). Universidad Ricardo Palma, Santiago de Surco. Recuperado de http://repositorio.urp.edu.pe/handle/URP/3036.

Secretaría de Salud. (28 de febrero de 2020). Comunicado técnico diario nuevo coronavirus en el mundo (covid-19). Recuperado de https://www.gob.mx/cms/uploads/attachment/file/571278/Comunicado_Tecnico_Di ario_COVID-19_2020.02.28.pdf.

Secretaría de Salud. (11 de marzo de 2020). Comunicado técnico diario nuevo coronavirus en el mundo (covid-19). Recuperado de https://www.gob.mx/cms/uploads/attachment/file/541487/Comunicado_Tecnico_Di ario_COVID-19_2020.03.11.pdf.

Secretaría de Salud. (30 de agosto de 2021). Datos abiertos bases históricas. Bases de datos históricas covid-19. Recuperado de https:/www.gob.mx/salud/documentos/datosabiertos-bases-historicas-direccion-general-de-epidemiologia.

Shi, H., Han, X., Jiang, N., Cao, Y., Alwalid, O., Gu, J., Fan, Y. and Zheng, C. (2020). Radiological findings from 81 patients with COVID-19 pneumonia in Wuhan, China: a descriptive study. The Lancet Infectious Diseases, 20(4), 425-434. Retrieved from https://doi.org/10.1016/S1473-3099(20)30086-4.

Suryahadi, A., Al Izzati, R. and Suryadarma, D. (2020). Estimating the Impact of COVID19 Outbreak on Poverty. Bulletin of Indonesian Economic Studies, 56(2), 175-192. Retrieved from https://doi.org/10.1080/00074918.2020.1779390. 

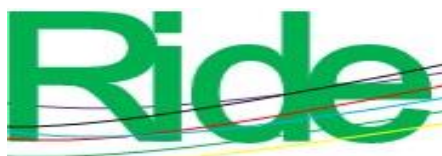

Revista Iberoamericana para la Investigación y el Desarrollo Educativo ISSN 2007 - 7467

Vilar, M., Pérez, V., Teruel, G., Alonso, A. and Pérez, R. (2020). Costing of actions to safeguard vulnerable Mexican households with young children from the consequences of COVID-19 social distancing measures. International Journal for Equity in Health, 19(1), 4-6. Retrieved from https://doi.org/10.1186/s12939-02001187-3.

Zar, H. J., Dawa, J., Bueno, G. and Castro, J. A. (2020). Challenges of COVID-19 in children in low- and middle-income countries. Paediatric Respiratory Reviews, 35, 70-74. Retrieved from https://doi.org/10.1016/j.prrv.2020.06.016. 


\begin{tabular}{|c|c|}
\hline Rol de Contribución & Autor (es) \\
\hline Conceptualización & Martha Jiménez García \\
\hline Metodología & $\begin{array}{l}\text { Martha Jiménez García «igual», Ma. De los Ángeles Martínez } \\
\text { Ortega «igual» }\end{array}$ \\
\hline Software & $\begin{array}{l}\text { Martha Jiménez García «igual», Pilar Gómez Miranda «igual», } \\
\text { Maria Elena Tavera Cortes «igual», Francisco Pérez Soto } \\
\text { «igual» }\end{array}$ \\
\hline Validación & $\begin{array}{l}\text { Martha Jiménez García «igual», Maria Elena Tavera Cortes } \\
\text { «igual» }\end{array}$ \\
\hline Análisis Formal & $\begin{array}{l}\text { Martha Jiménez García «igual», Maria Elena Tavera Cortes } \\
\text { «igual» }\end{array}$ \\
\hline Investigación & $\begin{array}{l}\text { Martha Jiménez García «igual», Pilar Gómez Miranda «igual», } \\
\text { Maria Elena Tavera Cortes «igual», Ma. De los Ángeles } \\
\text { Martínez Ortega «igual», Francisco Pérez Soto «igual» }\end{array}$ \\
\hline Recursos & Martha Jiménez García \\
\hline Curación de datos & $\begin{array}{l}\text { Martha Jiménez García «igual», Pilar Gómez Miranda «igual», } \\
\text { Maria Elena Tavera Cortes «igual», Ma. De los Ángeles } \\
\text { Martínez Ortega «igual», Francisco Pérez Soto «igual» }\end{array}$ \\
\hline $\begin{array}{l}\text { Escritura - Preparación del } \\
\text { borrador original }\end{array}$ & $\begin{array}{l}\text { Martha Jiménez García «igual», Pilar Gómez Miranda «igual», } \\
\text { Maria Elena Tavera Cortes «igual», Francisco Pérez Soto } \\
\text { «igual» }\end{array}$ \\
\hline Escritura - Revisión y edición & $\begin{array}{l}\text { Martha Jiménez García «igual», Ma. De los Ángeles Martínez } \\
\text { Ortega «igual» }\end{array}$ \\
\hline Visualización & $\begin{array}{l}\text { Martha Jiménez García «igual», Pilar Gómez Miranda «igual», } \\
\text { Maria Elena Tavera Cortes «igual», Ma. De los Ángeles } \\
\text { Martínez Ortega «igual» }\end{array}$ \\
\hline Supervisión & Martha Jiménez García \\
\hline Administración de Proyectos & Martha Jiménez García \\
\hline Adquisición de fondos & Martha Jiménez García \\
\hline
\end{tabular}

